\title{
Non-Covalent Interactions of N-(4-CarboxyPhenyl)Phthalimide with CNTs
}

\author{
Mehrnoush Molaeian $^{1}$, Asghar Davood $^{1}$, Mahmoud Mirzaei $^{2, 凶}$
}

Received: 10 March 2020 / Accepted: 14 March 2020 / Published Online: 16 March 2020

(C) SAMI Publishing Company (SPC) 2020

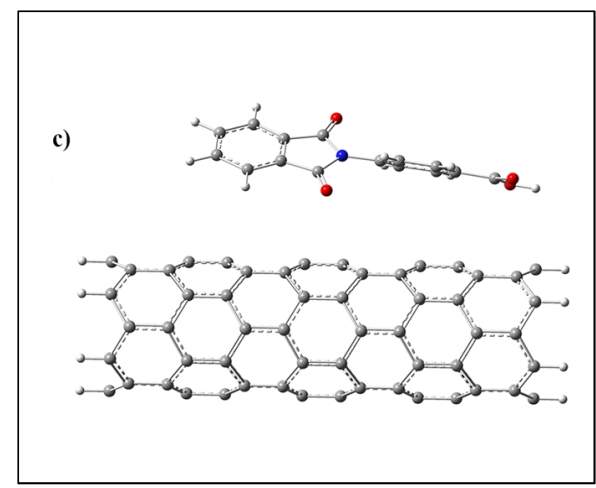

ABSTRACT
Non-covalent interactions of $\mathrm{N}$-(4-carboxyphenyl)phthalimide
(CPP) with carbon nanotubes (CNTs) have been investigated to see
the effects of interactions on the properties of CPP, which is a
medicinal compound. Two models of $(3,3)$ armchair and $(6,0)$ zigzag
CNTs have been considered in this work. All structures have been
optimized by density functional theory (DFT) calculations to
evaluate the corresponding properties. Moreover, quadrupole
coupling constants ( $\left.C_{Q}\right)$ have been evaluated at the atomic scale for
the optimized structures. The results yielded stabilized CPP@CNT
hybrids by effects of hybridization on the properties of both of CPP
and CNT counterparts. The CQ parameters also indicate that the
carbon atoms are very much important to detect the type of CNT
whereas other atoms showed almost the same effects at the same
situations. As a result, the CPP could be very well hybridized with
the CNT through non-covalent interacting system.

Keywords: N-(4-carboxyphenyl)phthalimide; CNT; Interaction; Hybrid; Density functional theory.

\section{Introduction}

Since the early days of carbon nanotubes (CNTs) discovery, they have been employed in various situations to explore their properties for specific applications [1-3]. In addition to the original CNT, several types of nanostructures have been developed for different purposes [4-11]. Covalent and non-covalent hybridizations of nanostructures have been done by other bioorganic, organic and inorganic counterparts to explore the effects on the properties of both hybridized sides [12-15]. One of the important roles of nanostructures is their expected applications in therapeutics, in which drug delivery purposes are among the most favorable ones [16-20]. To this aim, the properties of nanostructures should be carefully

\footnotetext{
$\triangle$ Corresponding author.

E-mail address: mdmirzaei@pharm.mui.ac.ir (M. Mirzaei)

${ }^{1}$ Department of Medicinal Chemistry, Faculty of Pharmaceutical Sciences, Tehran Islamic Azad Medical Sciences University, Tehran, Iran
}
${ }^{2}$ Biosensor Research Center, School of Advanced Technologies in Medicine, Isfahan University of Medical Sciences, Isfahan, Iran


characterized prior to assigning them for the application in living systems [21]. Besides experiments, computational chemistry approaches provide insightful methodologies to investigate the characteristics of complicated nanostructures [22]. These computations could be very much important to recognize differences in the properties of CNT tubular systems [23]. In this work, the computational chemistry approach has been used to explore the properties of noncovalent $\mathrm{N}$-(4-carboxyphenyl)phthalimide (CPP) and CNTs interacting systems of (Fig. 1).

CPP has anticonvulsant effects in the living systems but should still be improved to have higher efficacy during pharmacotherapy [24-29]. Since the mood disorders and mental diseases are very much common for the people of modern societies, careful attentions should be paid to improve the efficacy of such drugs for the related patients [30]. Therefore, hybridization with CNTs, as possible carriers for drug delivery processes, could be considered as one of the ways in this case. However, prior to any judgment, the properties of CPP@CNT hybrids should be recognized in order to see the effects of noncovalent interactions on the original properties of CPP. To this aim, this work has been done to recognize the mentioned properties at the atomic/molecular scales employing the computational chemistry approaches (Tables 1 and 2, Fig. 1). It is important to mention that theories and computers provided in silico environment to carefully characterize the properties of matters at the atomic/molecular scales besides the already well known in vitro and in vivo environments [31-35].

\section{Materials and Methods}

Within this work, we have calculated the minimal energies and properties for the investigated structures using the B3LYP exchange-correlation functional and the 6-31G* standard basis set as implemented in the Gaussian 98 package [36]. a)

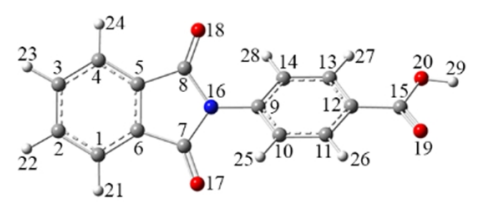

b)
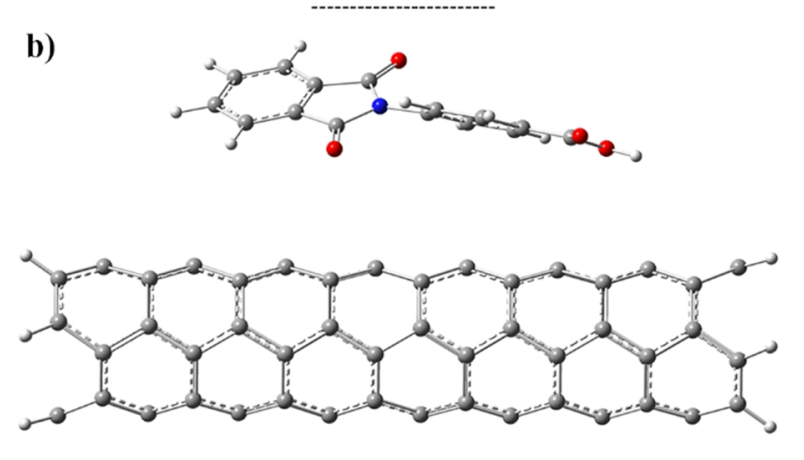

c)
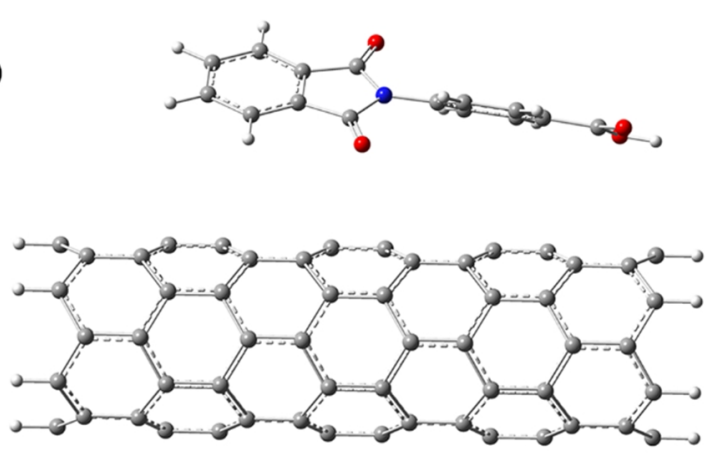

Fig. 1. a) The individual CPP, b) the hybrid of CPP@ $(3,3)$ CNT and c) the hybrid of CPP@ $(6,0)$ CNT.

The models of this work are N-(4carboxyphenyl)phthalimide (CPP) and representative $(3,3)$ armchair and $(6,0)$ zigzag carbon nanotubes (CNTs), in which their noncovalent interactions are investigated between CPP and CNTs; CPP@ $(3,3)$ and CPP@ $(6,0)$. The tubular tips of representative CNT models are saturated with hydrogen atoms to avoid any dangling effects to the structures [37, 38]. First, the isolated models have been allowed to fully relax during the optimization processes to achieve the structures corresponding to minimal energies. Afterwards, the optimized models have been combined together to make the hybrid structures of CPP@ $(3,3)$ and CPP@ $(6,0)$. For the hybrids, the pre-optimized geometries of CNT counterparts have been kept frozen to mimic the infinite-length nanotubes whereas those of CPP have been 
allowed to fully re-relax at the tubular surfaces. The results of optimizations indicated the final hybrid structures of CPP and CNTs according to the calculated minimal energies. In addition to the optimized structures, molecular properties have been also evaluated for both of isolated and hybrid systems including total energy $\left(E_{\mathrm{T}}\right)$, binding energy $\left(E_{\mathrm{B}}\right)$, energies for the highest occupied and the lowest unoccupied molecular orbitals (HOMO and LUMO), and dipole moment $\left(D_{\mathrm{M}}\right)$ (Table 1$)$. To clarify effects of CNTs on the properties of CPP, quadrupole coupling constants $\left(C_{Q}\right)$ have been evaluated for the atoms of optimized structures (Table 2). The effects to original electronic properties of atomic sites of interacting CPP in the hybrid structures are investigated by comparing the values of $C_{Q}$ in the isolated and hybrid systems. It is noted that $C_{Q}$ parameters indicate the electronic properties of individual atomic sites, which are helpful for atomic scale investigations of matters [39]. The exact magnitudes of $C_{Q}$ could be experimentally measured by nuclear quadrupole resonance (NQR) spectroscopy as an important technique in the category of nuclear magnetic resonance (NMR) [40]. Moreover, quantum chemical calculations could yield reliable atomic scale $C_{Q}$ properties for the investigating systems [41-50].

Table 1: The evaluated optimized properties ${ }^{*}$

\begin{tabular}{lllllll}
\hline Model & Formula & $\boldsymbol{E}_{\mathrm{T}} / \mathbf{k e V}$ & $\boldsymbol{E}_{\mathrm{B}} / \mathrm{eV}$ & $\boldsymbol{E}_{\text {Hомо }} / \mathrm{eV}$ & $\boldsymbol{E}_{\text {LUmo }} / \mathrm{eV}$ & $\boldsymbol{D}_{\mathrm{M}} /$ Debye \\
\hline CPP & $\mathrm{C}_{15} \mathrm{H}_{9} \mathrm{NO}_{4}$ & -25.381 & - & -6.691 & -2.469 & 4.702 \\
$\mathbf{( 3 , 3 )}$ & $\mathrm{C}_{96} \mathrm{H}_{12}$ & -99.721 & - & -4.263 & -2.622 & 0 \\
CPP@(3,3) & $\mathrm{C}_{111} \mathrm{H}_{21} \mathrm{NO}_{4}$ & -126.104 & 1002 & -4.253 & -2.618 & 3.960 \\
$\mathbf{( 6 , 0 )}$ & $\mathrm{C}_{96} \mathrm{H}_{12}$ & -99.728 & - & -3.802 & -3.152 & 0 \\
CPP@(6,0) & $\mathrm{C}_{111} \mathrm{H}_{21} \mathrm{NO}_{4}$ & -126.115 & 1006 & -3.805 & -3.168 & 3.210 \\
\hline
\end{tabular}

${ }^{*}$ See Fig. 1 for details.

\section{Results and Discussion}

The results of this work are divided into two parts based on the optimized properties (Table 1 ) and the $C_{Q}$ parameters (Table 2). The major question of this work is to investigate the effects of interactions on the properties of CPP at the surface of representative CNTs. This trend is aimed to see the effects of hybridizations on the initial properties of the loaded medicine on the CNT. As an important expected role for CNTs, they are willing to be used for drug deliveries but they showed so many unanswered questions for the purpose up to now [21]. In the current research, we would like to better clarify the possible interactions of CPP with CNTs through atomic/molecular scale quantum chemical calculations. As an advantage of computational chemistry, the chemical structures could be significantly investigated at the smallest sizes of one molecule or one atom, which are not easily achieved by experiments. Herein, the effort on this work has been dedicated to atomic/molecular scale investigations of properties for the CPP@CNT non-covalent interacting hybrid structures.

The structural models have been prepared in two sets of individual and hybrid systems in current work. For the individual system, there are three structures including CPP, $(3,3)$ and $(6,0)$ CNTs. The criterion of choosing the CNTs was the equality of atoms numbers in molecular formula, in which both CNTs have the same $\mathrm{C}_{96} \mathrm{H}_{12}$ stoichiometry. The optimization processes yielded the stable structures of individual systems showing that the $(6,0)$ CNT is slightly more stable than $(3,3)$ CNT with a difference magnitude of $7 \mathrm{eV}$ for total energies (Table 1 and Fig. 1). Studying ultra-small size nanotubes is an advantage of computational 
chemistry, which could not be easily done through experiments. After singular optimizations, the new structures have been combined together to make hybrid structures. The new optimization processes have been performed by fixing the geometries of CNT counterpart but re-relaxing the geometries of CPP at the surface of nanotube. It is important to note that fixing the geometries of CNTs is done to mimic the infinite-length nanotubes in the real states. Interestingly, the CPP@ $(6,0)$ has been viewed slightly more stable than CPP@ $(3,3)$ hybrid structure. Moreover, the binding energy for CPP@ $(6,0)$ is also better than CPP@ $(3,3)$ hybrid. To calculate the binding energies, the energy differences between the hybrid structure and its individual counterparts participating in the hybrid system have been considered. The energies for HOMO and LUMO levels reveal that the effects of hybridizations are detected by these electronic properties. From the singular to hybrid forms, the HOMO/LUMO levels will significantly change for the CPP but the changes for CNTs are in lower magnitudes. The HOMO/LUMO levels are different for the individual CNTs, in which their corresponding hybrids also show different values. The values of $D_{\mathrm{M}}$ are zero for both of individual CNTs; however, non-zero values for hybrids show the importance of biomolecular functionalization of CNTs for better dispersions in water media [21]. The value of $D_{\mathrm{M}}$ for individual CPP undergoes to lower values in the hybrids. As a concluding remark of this part, the optimization processes yielded the stabilized structures of non-covalent interacting CPP@CNT hybrids, in which both of $(3,3)$ and $(6,0)$ CNTs could be seen useful for the purpose.

Table 2: The evaluated $C_{Q} / \mathrm{MHz}$ parameters for CPP counterpart ${ }^{*}$

\begin{tabular}{|c|c|c|c|c|c|c|c|}
\hline Atom & CPP & CPP@(3,3) & CPP@(6,0) & Atom & CPP & CPP@(3,3) & CPP@(6,0) \\
\hline $1 \mathrm{C}$ & 2.364 & 2.396 & 2.412 & $16 \mathrm{~N}$ & 3.466 & 3.478 & 3.492 \\
\hline $2 \mathrm{C}$ & 2.294 & 2.298 & 2.286 & 170 & 9.862 & 9.849 & 9.868 \\
\hline $3 C$ & 2.293 & 2.280 & 2.259 & 180 & 9.857 & 9.849 & 9.828 \\
\hline $4 C$ & 2.362 & 2.349 & 2.331 & 190 & 8.836 & 8.863 & 8.782 \\
\hline $5 \mathrm{C}$ & 1.883 & 1.875 & 1.871 & 200 & 8.818 & 8.739 & 8.811 \\
\hline $6 C$ & 1.885 & 1.877 & 1.878 & $21 \mathrm{H}$ & 0.199 & 0.199 & 0.199 \\
\hline $7 C$ & 2.608 & 2.618 & 2.604 & $22 \mathrm{H}$ & 0.199 & 0.199 & 0.199 \\
\hline $8 \mathrm{C}$ & 2.610 & 2.614 & 2.622 & $23 \mathrm{H}$ & 0.199 & 0.199 & 0.199 \\
\hline $9 \mathrm{C}$ & 2.356 & 2.339 & 2.341 & $24 \mathrm{H}$ & 0.199 & 0.199 & 0.199 \\
\hline $10 \mathrm{C}$ & 1.957 & 1.955 & 1.958 & $25 \mathrm{H}$ & 0.202 & 0.202 & 0.202 \\
\hline $11 \mathrm{C}$ & 2.501 & 2.495 & 2.485 & $26 \mathrm{H}$ & 0.198 & 0.198 & 0.199 \\
\hline $12 \mathrm{C}$ & 1.471 & 1.488 & 1.413 & $27 \mathrm{H}$ & 0.199 & 0.199 & 0.200 \\
\hline $13 \mathrm{C}$ & 2.406 & 2.416 & 2.406 & $28 \mathrm{H}$ & 0.202 & 0.202 & 0.201 \\
\hline $14 \mathrm{C}$ & 1.941 & 1.949 & 1.957 & $29 \mathrm{H}$ & 0.278 & 0.278 & 0.279 \\
\hline $15 \mathrm{C}$ & 2.812 & 2.664 & 2.858 & & & & \\
\hline
\end{tabular}

${ }^{*}$ See Fig. 1 for details.

The atomic scale properties of CPP in the individual and hybrid forms are investigated by the values of $C_{Q}$ parameters to see the effects of interactions on the electronic properties of CPP at the CNTs surfaces. The results are discussed in the atomic types as listed in Table 2 . The results indicate that the carbon atoms detect different electronic environments at the surfaces of $(3,3)$ and $(6,0)$ CNTs. This trend is very much important because of advantage of evaluating $C_{Q}$ atomic scale properties in addition to the molecular properties from the optimization processes. As 
could be seen by the panels $b$ and $c$ of Fig. 1, the position of CPP is slightly different at the surfaces of the $(3,3)$ and $(6,0)$ CNTs. Therefore, the $C_{Q}$ atomic scale properties indicate different parameters for the CPP at two hybrid forms. There is only one nitrogen atom in CPP, in which it still shows effects of CNT types at its electronic environment. Moreover, the effects of interactions could be seen by comparing the results of nitrogen atom of CPP individual and hybrid systems. These effects are also seen for the oxygen atoms of CPP from the individual system to the hybrid system. It is worth noting that the magnitude of $C_{Q}$ is dependent on the atomic number, in which the oxygen atom shows the largest magnitude of $C_{Q}$ among the investigated atoms. Since the hydrogen atom has the smallest number of electrons, its changes are at the lowest level in comparison with the other atoms. As a concluding remark of this part, the $C_{Q}$ atomic scale parameters could very well shoe details of investigating systems to clarify what is happening inside the molecular structures.

\section{Conclusion}

The effects of non-covalent interactions on the properties of CPP have been investigated at the surfaces of $(3,3)$ and $(6,0)$ CNTs based on computational chemistry approaches. By examining the obtained results, some trends could

\section{References}

1. lijima S. Helical microtubules of graphitic carbon. Nature 1991;354:56-58.

2. Yang Z, Tian J, Yin Z, Cui C, Qian W, Wei F. Carbon nanotube-and graphene-based nanomaterials and applications: A review. Carbon 2019;141:467-480.

3. Barnard AW, Zhang M, Wiederhecker GS, Lipson $M$, McEuen PL. Real-time vibrations of a carbon nanotube. Nature 2019;566:89-93.

4. Mirzaei M. Calculation of chemical shielding in C-doped zigzag BN nanotubes. Monatsh. Chem. 2009;140:12751278.

5. Mirzaei M, Mirzaei M. The B-doped SiC nanotubes: A computational study. J. Mol. Struct. THEOCHEM 2010;953:134-138. be concluded. First, the CPP could be very well stabilized at the surface of CNTs, in which both of $(3,3)$ and $(6,0)$ models could work for this purpose. Second, the molecular properties could show the effects of interactions on the properties of CPP from individual to hybrid system, in which the HOMO/LUMO properties could be seen as useful parameters. Third, the values of $D_{\mathrm{M}}$ could show the importance of biomolecular functionalization of CNTs for better dispersion properties in water media. Fourth, the $C_{Q}$ atomic scale properties indicated significant effects of CNT types on the electronic properties of carbon atoms of CPP whereas this trend was not seen for the nitrogen and oxygen atoms. Fifth, all the $C_{Q}$ atomic scale properties shoed the effects of non-covalent interactions on the properties of CPP, in which higher and lower changes have been seen based on the CNT types or the atomic types. Finally, the hybrid models of CPP@CNT could be considered as the non-covalent interacting systems for different purposes of applications, especially for the living systems.

\section{Acknowledgements}

The authors acknowledge the supports of this work by the research council of Islamic Azad University under the grant number 2755, which has been done as the pharmacy doctoral thesis of M. Molaeian.
6. Mirzaei M. Carbon doped boron phosphide nanotubes: a computational study. J. Mol. Model. 2011;17:89-96.

7. Mirzaei M. Density functional study of defects in boron nitride nanotubes. Z. Phys. Chem. 2009;223:815-823.

8. Mirzaei M, Hadipour NL, Seif A, Giahi M. Density functional study of zigzag $B N$ nanotubes with equivalent ends. Physica E 2008;40:3060-3063.

9. Mirzaei M, Mirzaei M. The C-doped AIP nanotubes: A computational study. Solid State Sci. 2011;13:244-250.

10. Mirzaei M. A computational NMR study of boron phosphide nanotubes. Z. Naturforsch. A 2010;65:844848.

11. Mirzaei M. The NMR parameters of the SiC-doped BN nanotubes: a DFT study. Physica E 2010;42:1954-1957. 
12. Ebrahim-Habibi MB, Ghobeh M, Mahyari FA, RafiiTabar H, Sasanpour P. An investigation into noncovalent functionalization of a single-walled carbon nanotube and a graphene sheet with protein G: A combined experimental and molecular dynamics study. Sci. Rep. 2019;9:1-8.

13. Park M, Lee $H$, Jang JU, Park JH, Kim CH, Kim SY, Kim J. Phenyl glycidyl ether as an effective noncovalent functionalization agent for multiwalled carbon nanotube. Compos. Sci. Technol. 2019;177:96-102.

14. Mirzaei M, Kalhor HR, Hadipour NL. Covalent hybridization of CNT by thymine and uracil: $A$ computational study. J. Mol. Model. 2011;17:695-699.

15. Mirzaei M, Yousefi M. Computational studies of the purine-functionalized graphene sheets. Superlat. Microstruct. 2012;52:612-617.

16. Kumar RM, Rajesh K, Haldar S, Gupta P, Murali K, Roy $P$, Lahiri D. Surface modification of CNT reinforced UHMWPE composite for sustained drug delivery. J. Drug Delivery Sci. Technol. 2019;52:748-759.

17. Sheikhi M, Shahab S, Khaleghian M, Ahmadianarog $M$, Azarakhshi F, Kumar R. Investigation of the adsorption rubraca anticancer drug on the CNT $(4,4-8)$ nanotube as a factor of drug delivery. Cur. Mol. Med. 2019;19:473-486.

18. Li H, Sun X, Li Y, Li B, Liang C, Wang H. Preparation and properties of carbon nanotube (Fe)/hydroxyapatite composite as magnetic targeted drug delivery carrier. Mater. Sci. Engin. C 2019;97:222-229.

19. Dong P, Rakesh KP, Manukumar HM, Mohammed YH, Karthik CS, Sumathi S, Mallu P, Qin HL. Innovative nanocarriers in anticancer drug delivery-a comprehensive review. Bioorg. Chem. 2019;85:325-336.

20. Mirzaei M. Effects of carbon nanotubes on properties of the fluorouracil anticancer drug: DFT studies of a CNT-fluorouracil compound. Int. J. Nano Dimens. 2013;3:175-179.

21. Panwar N, Soehartono AM, Chan KK, Zeng S, Xu G, Qu $J$, Coquet $P$, Yong KT, Chen X. Nanocarbons for biology and medicine: sensing, imaging, and drug delivery. Chem. Rev. 2019;119:9559-9656.

22. Srivastava D, Menon M, Cho K. Computational nanotechnology with carbon nanotubes and fullerenes. Comput. Sci. Engin. 2001;3:42-55.

23. Belin T, Epron F. Characterization methods of carbon nanotubes: a review. Mater. Sci. Engin. B 2005;119:105-118.

24. Kumar V, Banker GS. Incompatibility of polyvinyl acetate phthalate with benzocaine: Isolation and characterization of 4-phthalimidobenzoic acid ethyl ester. Int. J. Pharm. 1992;79:61-65.

25. Liang ZP, Li J, Huang BY. 4-Phthalimidobenzoic acid N, N-dimethylformamide solvate. Acta Cryst. E 2006;62:4761-4762.

26. Iman M, Fakhari S, Jahanpanah M, Naderi N, Davood A. Design and synthesis of 4-flurophthalimides as potential anticonvulsant agents. Iran. J. Pharm. Res. 2018;17:896-905.
27. Vamecq J, Bac P, Herrenknecht $C$, Maurois $P$, Delcourt $P$, Stables JP. Synthesis and anticonvulsant and neurotoxic properties of substituted N-phenyl derivatives of the phthalimide pharmacophore. J. Med. Chem. 2000;43:1311-1319.

28. Lovitt JI, Hawes CS, Gunnlaugsson T. Crystallographic studies of 2-picolyl substituted naphthalene diimide and bis-phthalimide ligands and their supramolecular coordination chemistry. CrystEngComm. 2019;21:207217.

29. Singh RB, Singh GK, Chaturvedi K, Kumar D, Singh SK, Zaman MK. Design, synthesis, characterization, and molecular modeling studies of novel oxadiazole derivatives of nipecotic acid. Med. Chem. Res. 2018;27:137-152.

30. Patel N, Viguera AC, Baldessarini RJ. Mood-stabilizing anticonvulsants, spina bifida, and folate supplementation: commentary. J. Clin. Psychopharm. 2018;38:7-10.

31. Soleimani M, Mirzaei M, Mofid MR, Khodarahmi G, Rahimpour SF. Lactoperoxidase inhibition by tautomeric propylthiouracils. Asian J. Green Chem. 2020;4:1-0.

32. Alidoosti ZS, Mirzaei M. Comparative examination of moclobemide, tranylcypromine, phenelzine and isocarboxazid for monoamine oxidase-A inhibition. Adv. J. Chem. B 2019;1:23-28.

33. Esfahani AN, Mirzaei M. Flavonoid derivatives for monoamine oxidase-A inhibition. Adv. J. Chem. B 2019;1:17-22.

34. Ozkendir OM, Mirzaei M. Alkali metal chelation by 3hydroxy-4-pyridinone. Adv. J. Chem. B 2019;1:10-6.

35. Nazemi H, Mirzaei M, Jafari E. Antidepressant activity of curcumin by monoamine oxidase-A inhibition. J. Adv. Chem. B 2019;1:3-9.

36. Frisch MJ, Trucks GW, Schlegel HB, Scuseria GE, Robb MA, Cheeseman JR, Zakrzewski VG, Montgomery Jr JA, Stratmann RE, Burant JC, Dapprich S, et al. Gaussian 98, Revision A. 7. Pittsburgh, PA: Gaussian. Inc. Computer Program. 1998.

37. Bodaghi A, Mirzaei M, Seif A, Giahi M. A computational NMR study on zigzag aluminum nitride nanotubes. Physica E 2008;41:209-212.

38. Mirzaei M, Meskinfam M. Computational studies of effects of tubular lengths on the NMR properties of pristine and carbon decorated boron phosphide nanotubes. Solid State Sci. 2011;13:1926-1930.

39. Wu G. Recent developments in solid-state nuclear magnetic resonance of quadrupolar nuclei. Biochem. Cell Biol. 1998;76:429-442.

40. Shen J, Terskikh V, Wang X, Hung I, Gan Z, Wu G. A quadrupole-central-transition $170 \mathrm{NMR}$ study of nicotinamide. J. Phys. Chem. B 2018;122:4813-4820.

41. Mirzaei M, Hadipour NL. Study of hydrogen bonds in 1methyluracil by DFT calculations of oxygen, nitrogen, and hydrogen quadrupole coupling constants and isotropic chemical shifts. Chem. Phys. Lett 2007;438:304-307. 
42. Mirzaei M, Elmi F, Hadipour NL. A systematic investigation of hydrogen-bonding effects on the 170, $14 \mathrm{~N}$, and $2 \mathrm{H}$ nuclear quadrupole resonance parameters of anhydrous and monohydrated cytosine crystalline structures: a density functional theory study. J. Phys. Chem. B 2006;110:10991-10996.

43. Bagheri Z, Mirzaei M, Hadipour NL, Abolhassani MR. Density functional theory study of boron nitride nanotubes: calculations of the $\mathrm{N}-14$ and B-11 nuclear quadrupole resonance parameters. J. Comput. Theor. Nanosci. 2008;5:614-618.

44. Mirzaei M, Hadipour NL, Abolhassani MR. Influence of C-doping on the B-11 and N-14 quadrupole coupling constants in boron-nitride nanotubes: A DFT study. $Z$. Naturforsch. A 2007;62:56-60.

45. Mirzaei M, Hadipour NL. A computational NQR study on the hydrogen-bonded lattice of cytosine-5-acetic acid. J. Comput. Chemi. 2008;29:832-838.

46. Mirzaei M, Hadipour NL, Ahmadi K. Investigation of C$\mathrm{H} . . . \mathrm{O}=\mathrm{C}$ and $\mathrm{N}-\mathrm{H} . . . \mathrm{O}=\mathrm{C}$ hydrogen-bonding interactions in crystalline thymine by DFT calculations of $0-17, \mathrm{~N}-14$ and H-2 NQR parameters. Biophys. Chem. 2007;125:411-415.

47. Behzadi H, Hadipour NL, Mirzaei M. A density functional study of $170,14 \mathrm{~N}$ and $2 \mathrm{H}$ electric field gradient tensors in the real crystalline structure of $\alpha$ glycine. Biophys. Chem. 2007;125:179-183.

48. Samadi Z, Mirzaei M, Hadipour NL, Khorami SA. Density functional calculations of oxygen, nitrogen and hydrogen electric field gradient and chemical shielding tensors to study hydrogen bonding properties of peptide group $(\mathrm{O}=\mathrm{C}-\mathrm{NH})$ in crystalline acetamide. J. Mol. Graph. Model. 2008;26:977-981.

49. Harismah K, Mirzaei M, Moradi R. DFT studies of single lithium adsorption on coronene. Z. Naturforsch. A 2018;73:685-691.

50. Partovi T, Mirzaei M, Hadipour NL. The $\mathrm{C}-\mathrm{H} \cdots \mathrm{O}$ hydrogen bonding effects on the 170 electric field gradient and chemical shielding tensors in crystalline 1methyluracil: A DFT study. Z. Naturforsch. A 2006;61:383-388.

How to cite this article: Molaeian M, Davood A, Mirzaei M. Non-Covalent Interactions of N-(4CarboxyPhenyl)Phthalimide with CNTs. Adv. J. Chem. B. 2020;2(1):39-45. doi: 10.33945/SAMI/AJCB.2020.1.7 